\section{USE OF THE COMBINED POSITIVE SCORE (CPS) WITH THE COMPANION DIAGNOSTIC PD-L1 IHC 22C3 PHARMDX PROVIDES PRECISE EVALUATION OF PD-L1 EXPRESSION ACROSS MULTIPLE TUMOR INDICATIONS AND CUTOFFS}

Francisco Ponce*, Stephanie Hund, Lindsay Peltz, Chris La Placa, Monika Vilardo, Brittany Watts, Siena Tabuena-Frolli, Grant Toland, Alex Posch, Jay Milo, Karina Kulangara, Angeliki Apostolaki. Agilent Technologies, Carpinteria, CA, USA

Background The Combined Positive Score (CPS) ${ }^{1}$ algorithm includes tumor and immune cells for determination of Programed Death-Ligand 1 (PD-L1) protein expression in tumor tissues and has been analytically and clinically validated for use with PD-L1 IHC 22C3 pharmDx across multiple indications and cutoffs. ${ }^{2}$ PD-L1 22C3 IHC pharmDx is a qualitative immunohistochemical assay using anti-PD-L1, Clone 22C3 to detect PD-L1 in formalin-fixed, paraffin-embedded (FFPE) tumor tissues using Autostainer Link 48. PD-L1 IHC 22C3 pharmDx is FDA-approved as an aid in identifying patients for treatment with KEYTRUDA ${ }^{\circledR}$ for six tumor indications at clinically validated CPS diagnostic cutoffs ${ }^{2}$ : gastric or gastroesophageal junction (GC/GEJ) adenocarcinoma (CPS $\geq 1$ ), cervical cancer (CPS $\geq 1$ ), urothelial carcinoma (CPS $\geq 10$ ), head and neck squamous cell carcinoma (HNSCC) (CPS $\geq 1$ ), esophageal squamous cell carcinoma (ESCC) $(\mathrm{CPS} \geq 10)^{3}$, and triple-negative breast cancer (TNBC) (CPS $\geq 10$ ).

Methods Precision of PD-L1 IHC 22C3 pharmDx using CPS was assessed for all six indications at the corresponding clinically validated diagnostic cutoffs and at additional exploratory cutoffs under normal, day-to-day testing conditions. Precision testing included Combined Precision (inter-instrument/operator/ run (day)), Intra-Run Repeatability, and Observer (inter-/intra-) Scoring Reproducibility studies. FFPE specimens were stained with PD-L1 IHC 22C3 pharmDx and scored using CPS as described in the package insert. ${ }^{2}$ Four CPS cutoffs were evaluated: CPS $\geq 1$ (GC/GEJ, urothelial carcinoma, ESCC, cervical cancer, HNSCC, TNBC), CPS $\geq 10$ (GC/GEJ, urothelial carcinoma, ESCC, TNBC), CPS $\geq 20$ (HNSCC), and CPS $\geq$ 50 (HNSCC). Data were analyzed using negative percent agreement (NPA), positive percent agreement (PPA), and overall agreement (OA) with two-sided 95\% percentile bootstrap confidence intervals (CIs) based on PD-L1 binary status at the applicable cutoff(s). For each study, data from each CPS cutoff-indication pair were individually analyzed. Meta-analyses were also performed by pooling data from all indications per (i) study and cutoff, and (ii) per study for all tested cutoffs.

Results Nearly all agreement analyses (142/144) for each CPS cutoff-indication pair showed NPA/PPA/OA point estimates $(\mathrm{PE}) \geq 90 \%$ and CI lower bounds (CILB) $\geq 85 \%$. Meta-analyses showed $\mathrm{PE} \geq 90 \%$ for $\mathrm{NPA} / \mathrm{PPA} / \mathrm{OA}$ and $\mathrm{CILB} \geq 85 \%$ per study and cutoff, and per study for all tested cutoffs. Discordant comparisons accounted for $<5 \%$ of total comparisons performed for each study type.

Conclusions CPS used with PD-L1 IHC 22C3 pharmDx provides precise evaluation of PD-L1 expression across multiple tumor indications and cutoffs under normal, day-to-day testing conditions.

Acknowledgements We thank the IUSCC Cancer Center at Indiana University School of Medicine, for the use of the Tissue Procurement and Distribution Core, which provided Dako North America, Inc. service.The data and biospecimens used in this project were provided by US Biolab (Gaithersburg, MD, USA), Sofia Bio LLC (New York, NY, USA), Contract
Research Ltd (Charlestown, Nevis), and Centre Hospitalier Universitaire (CHU) de Nice (Nice, France) with appropriate ethics approval and through Trans-Hit Biomarkers Inc. Tissue samples were provided by the Cooperative Human Tissue Network which is funded by the National Cancer Institute. Other investigators may have received specimens from the same subjects. Tissue samples supplied by BioIVT (Hicksville, NY, USA).

Trial Registration N/A

\section{REFERENCES}

1. $\quad$ PPS $=(\#$ PD-L1 staining cells (tumor cells, lymphotcytes, macrophages))/(Total \# viable tumor cells $) \times 100$

2. PD-L1 IHC 22C3 pharmDx [Instructions for Use]. Available at: www.agilent.com/ library/eifu. Code SK006. Accessed July 2, 2021

3. ESCC was analytically validated as a subtype of esophageal cancer [2].

\section{Ethics Approval N/A}

Consent N/A

http://dx.doi.org/10.1136/jitc-2021-SITC2021.060 